\title{
DUALITY IN CROSSED PRODUCTS AND VON NEUMANN ALGEBRAS OF TYPE III
}

\author{
BY MASAMICHI TAKESAKI \\ Communicated by Jacob Feldman, December 1, 1972
}

In this paper, we announce further results succeeding to the previous papers [2] and [3]. Suppose $\mathscr{M}$ is a von Neumann algebra and $G$ an abelian locally compact group. Let $\sigma: t \in G \rightarrow \sigma_{t} \in \operatorname{Aut}(\mathscr{M})$ be a continuous homomorphism in the sense that for each $x \in \mathscr{M}$, the map: $t \in \boldsymbol{R} \rightarrow \sigma_{t}(x) \in \mathscr{M}$ is $\sigma$-weakly continuous. We construct the crossed product $\mathscr{M} \otimes_{\sigma} G$ of $\mathscr{M}$ by $G$ with respect to $\sigma$. In [2], we have shown that there is a canonical dual action $\theta$ of the dual group $\hat{G}$ on $\mathscr{M} \otimes_{\sigma} G$ so that $\left(\mathscr{M} \otimes_{\sigma} G\right) \otimes_{\theta} \hat{G} \cong \mathscr{M} \otimes \mathscr{L}\left(L^{2}(G)\right)$, where $\mathscr{L}\left(L^{2}(G)\right)$ means, of course, the algebra of all bounded operators on the Hilbert space $L^{2}(G)$ of all square integrable functions on $G$ with respect to the Haar measure of $G$.

THEOREM 1. (i) If $H$ is a closed subgroup of $G$, then $\mathscr{M} \otimes_{\sigma} H$ is canonically imbedded in $\mathscr{M} \otimes_{\sigma} G$ and $\left\{p \in \hat{G}: \theta_{p}(x)=x\right.$ for every $\left.x \in \mathscr{M} \otimes_{\sigma} H\right\}$ is precisely the annihilator $H^{\perp}$ of $H$.

(ii) If $\hat{H}$ is a closed subgroup of $\hat{G}$, then the fixed point subalgebra $\left(\mathscr{M} \otimes_{\sigma} G\right)^{\hat{H}}$ of $\mathscr{M} \otimes_{\sigma} G$ under $\theta(\hat{H})$ is precisely $\mathscr{M} \otimes_{\sigma} H$, with $H$ the annihilator $\hat{H}^{\perp}$ of $\hat{H}$ in $G$.

We apply this theorem to the structure of von Neumann algebras of type III. In [3], we showed that for a von Neumann algebra $\mathscr{M}$ of type III, there exists uniquely a semifinite von Neumann algebra $\mathscr{M}_{0}$ equipped with a one parameter automorphism group $\left\{\theta_{t}\right\}$ such that $\mathscr{M} \cong \mathscr{M}_{0} \otimes_{\theta} \boldsymbol{R}$ and the action $\sigma_{t}$ of $\boldsymbol{R}$ on $\mathscr{M}$, which is dual to $\theta$, is the modular automorphism group associated with the faithful semifinite normal weight $\varphi$ which is canonically constructed from a trace $\tau$ on $\mathscr{M}_{0}$ with $\tau \cdot \theta_{t}=e^{t} \tau$, $t \in \boldsymbol{R}$. The above theorem implies immediately the following result.

COROLlaRY 2. Imbedding canonically $\mathscr{M}_{0}$ into $\mathscr{M}=\mathscr{M}_{0} \otimes{ }_{\theta} \boldsymbol{R}, \mathscr{M}_{0}$ is precisely the centralizer of the weight $\varphi$.

COROLlaRY 3. The center $\mathscr{Z}$ of $\mathscr{M}$ is precisely the fixed point subalgebra of the center $\mathscr{Z}_{0}$ of $\mathscr{M}_{0}$ under the action $\left\{\theta_{t}: t \in \boldsymbol{R}\right\}$.

We now consider a factor $\mathscr{M}$ of type III with separable predual $\mathscr{M}_{*}$. Making use of measure theoretic arguments, which are partly due to

AMS (MOS) subject classifications (1970). Primary 46L10. 
H. A. Dye, we can show the following:

THEOREM 4. If $\mathscr{M}$ is a factor of type III with separable predual $\mathscr{M}_{*}$, then $\mathscr{M}_{0}$ must be of type $\mathrm{II}_{\infty}$.

Making use of further ergodic theory type arguments, which we owe partly to T. Liggett, we can show the following:

THEOREM 5. If $\mathscr{M}$ is a factor of type III with separable predual, for $\mathscr{M}$ to be of type III $_{1}$ in the sense of Connes (i.e., $\left.S(\mathscr{M})=\boldsymbol{R}_{+}\right)$it is necessary and sufficient that $\mathscr{M}_{0}$ is a factor of type $\mathrm{II}_{\infty}$.

COROLlaRY 6. If $\mathscr{M}$ is a factor of type $\mathrm{III}_{1}$ with separable predual, then for $T>0$ the crossed product $\mathscr{M} \otimes_{\varphi}(T Z)$ of $\mathscr{M}$ by the closed subgroup $T Z$ of $\boldsymbol{R}$ with respect to the modular automorphism group $\sigma_{t}^{\varphi}$ associated with a faithful semifinite normal weight $\varphi$ is a factor of type III $_{\kappa}$ with $\kappa=e^{-2 \pi / T}$.

\section{REFERENCES}

1. A. Connes, Une classification de facteurs de type. III (to appear).

2. M. Takesaki, Dualité dans les produits croisés des algèbres de vom Neumann (to appear).

3. - Algèbres de von Neumann proprement infinies et produits croisés, C.R. Acad. Sci. Paris, Ser A 276 (1973), 125-127.

Department of Mathematics, University of California, los Angeles, California 90024 